ISASE2020

\author{
Concept of Artificial Kansei \\ - Challenge to New Artificial Intelligence- \\ Hisao Shiizuka * and Shigaku Tei ** \\ * SKEL, Shiizuka Kansei Engineering Laboratory, Tokyo, Japan \\ ** The University of Aizu, Fukushima, Japan
}

\begin{abstract}
Our purpose in this paper is to propose the concept of "artificial Kansei/sensibility". In recent years, artificial intelligence (AI) has been the focus of many people, but here we propose the basic concept of "artificial Kansei/sensibility" as a superordinate concept of artificial intelligence (AI). AI is good at deductive and inductive reasoning. Deep learning and machine learning are often performed based on deductive or inductive reasoning. Furthermore, AI cannot deal with abduction. The concept of artificial Kansei/sensibility proposed in this paper provides a methodology that can handle abduction and transilient inference, so that our new concept will extend current AI technology to treat GAN(Generative Adversarial Networks) .
\end{abstract}

Keywords: Artificial/Affective Kansei, Inference, Innovation tetra, GAN, Kansei/Affective Communication

\section{TARGET OF OUR INTEREST}

Recently, the concept of artificial intelligence (AI) is been emerging, but we are interested in artificial Kansei/sensibility, which is considered to be a superordinate concept of AI. As is well known, when we make inferences to explain something, there are deductive and inductive inferences, as shown in Fig.(a). Deduction is valid to elicit a hypothesis, it is impossible to incorporate new information. Induction is not complete intended to cover the outcome is never all of the analysis, which has a problem that another way is found always to investigate the subject. Charles Sanders Peirce was argued that the inference to generate a new idea is only abduction, as shown in Fig.1(b). The abduction is a reasoning method that first observe, then it proceeds on the hypothesis which may be true.

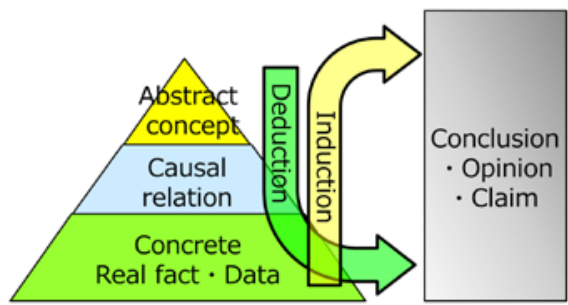

(a) Deduction and Induction are opposite relation each other

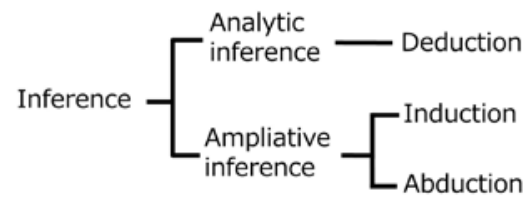

(b) Deduction, Induction and Abduction

Fig.1 Structure of inference
Inference can be broadly divided into "analytic inference” and "ampliative inference”, as shown in Fig.2. Here we add "transilient" as inference derived from amplificative inference. This transilient is completely free of thought. In other words, transilient is the idea of jumping freely across boundaries.

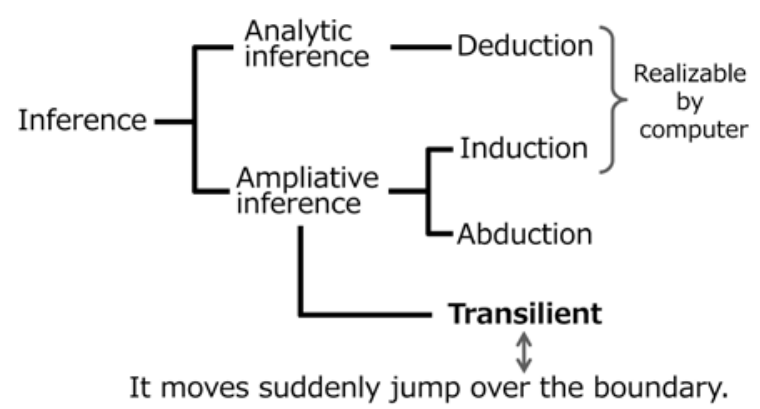

Fig.2 New Human Intellectual Life.

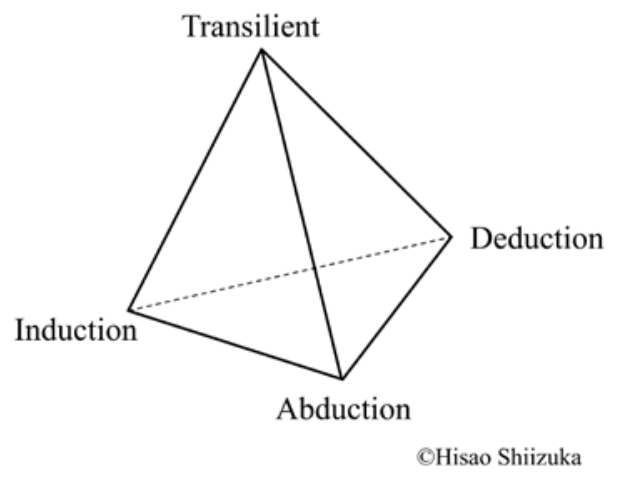

Fig.3 Innovation tetra

Here, Fig. 3 shows the deduction, induction, abduction, and transilient associated with each vertex of the "triangular pyramid". This is called "Innovation tetra", 
which is defined by Shiizuka [1][2].

Now, our interest is "can computers do abduction?" Today's AI has the power to perform deductive and inductive processing. However, with AI, abduction-like processing will not be possible at present. Here, we propose the concept of artificial sensibility which enables abduction and transilient.

\section{INNOVATION TETRAAND PORTFOLIO}

Figure 4 shows the four types of the innovation breakthrough [1][2]. In the case of these four, the last finish of reasoning can be seen that the deduction is responsible.

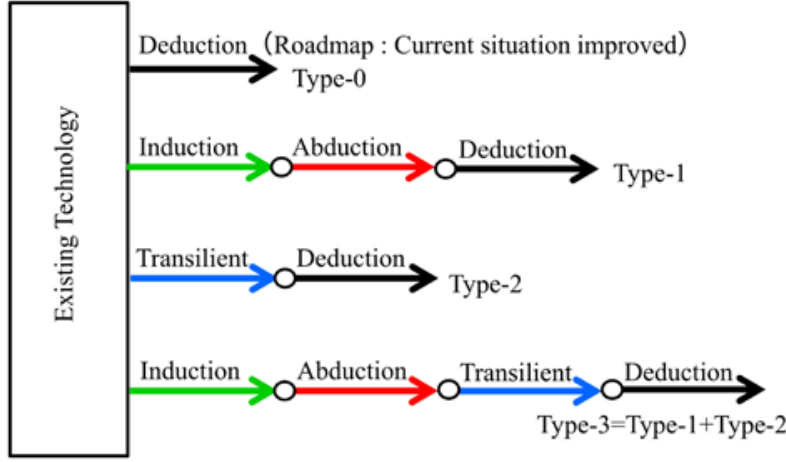

Fig.4 Innovation portfolio consisting of four types [1].

Innovation engineering is based on the elements of innovation portfolio. It is an object of risk minimization and profit maximization innovation. Innovation portfolio is that the combination selection in innovation of each element shown in Fig. 3. If we attempt to innovation, it must be promoted in view of both sides of the "innovation and advantage", and "reality and future potential". It is selected considering the appropriate combination is important in the efficiency of innovation. Concept of combination selection in this innovation is the same concept as that incorporated in the fields of finance and marketing. It may be determined by engineering methods the optimal strategy of the combination of elements (each type) in the innovation strategy.

Table 1 Shape of each type in Fig.4

\begin{tabular}{|c|c|}
\hline Type & Shape \\
\hline 0 \\
(Point) & $\bullet$ Deduction \\
\hline $\begin{array}{c}1 \\
\text { (Surface) }\end{array}$ & Transilient \\
\hline 2 \\
(Line)
\end{tabular}

Table 1 shows the representation of the innovation portfolio in a concrete form. Innovation-tetra is associating to each vertex of a triangular pyramid the four elements of the innovation portfolio, as shown in Fig.3. It is possible to determine the power set of four elements of the innovation-tetra, as shown in Fig.5.

The structure of innovation tetra for making inferences that lead to breakthrough is shown by Shiizuka [2]. It is shown that undirected graphs mean a bi-directional, that is, it indicates that the inference is possible from either side.

It must be pointed out the following to keep in mind when performing the innovation-related research, including the affective and sensibility subjects. That is to say, it is important to know the position and perspective of research which we have done.

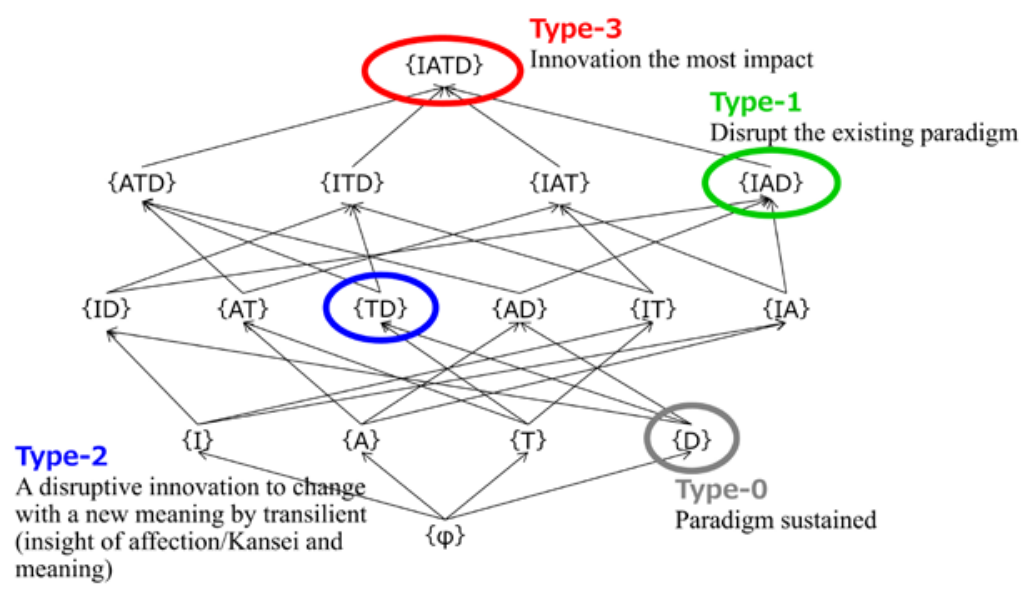

Fig.5 Power set of the innovation tetra described in Fig.3. 


\section{KANSEI/AFFECTIVE TURING TEST}

We assume that three plyers $\mathrm{A}, \mathrm{B}$, and $\mathrm{C}$ are having conversations in three rooms using "characters" via computers as shown in Fig.6. Next, player A is replaced with a computer as shown in Fig.7.

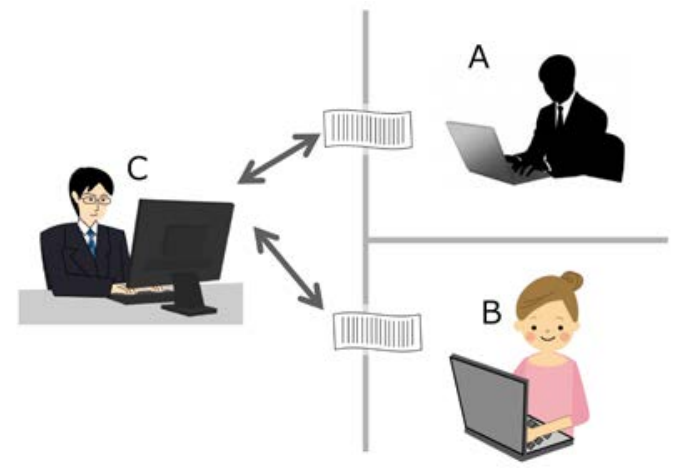

Fig.6 Both of A and B are human.

The "standard interpretation" of the Turing test, in which player $\mathrm{C}$, the interrogator, is given the task of trying to determine which player - A or B - is a computer and which is a human. The interrogator is limited to using the responses to written questions to make the determination.

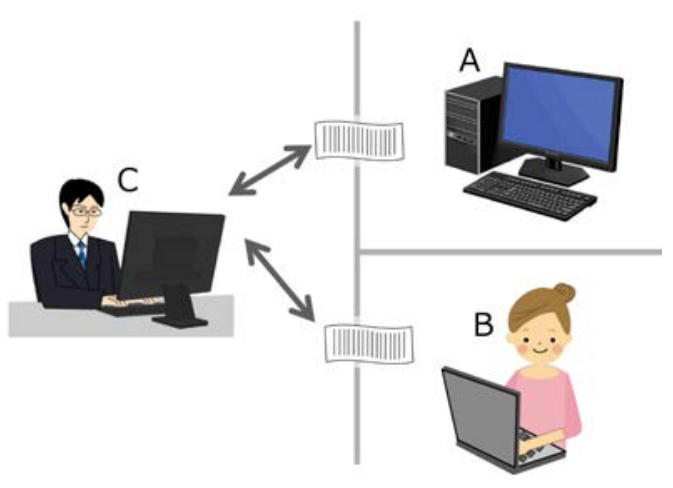

Fig.7 The standard interpretation of the Turing test.

At this time, when player $\mathrm{C}$ (the interrogator) does not know which of player B or computer $\mathrm{A}$ is a human, computer $\mathrm{A}$ is said to have intelligence.

Next, let's think about "affective communication" between humans. Because human communication includes ambiguous parts, it will be necessary to consider affective communication in consideration of ambiguity. It goes without saying that it is a very important thing that only humans can do. As shown in Fig. 8, what the sender wants to send now is a part where the arrow is "clear" and a part where the arrow is "blurred" around it. The former is a so-called "crisp content" that the sender can concretely think and specify. The latter is a "fuzzy" content that is vague and cannot be stated explicitly, and that one wants to rely on suggestion.

Therefore, the expressed information also has clear arrows (crisp information) and blurred portions (fuzzy information). Then, the recipient receives and interprets this information, but what the recipient understands is also divided into "clear parts" and "bleeding parts”. The former is a "crisp understanding" in which the sender's information is grasped verbatim and specifically, and the latter is a "fuzzy understanding" in which the recipient himself / herself interprets the information creatively and spectacularly.

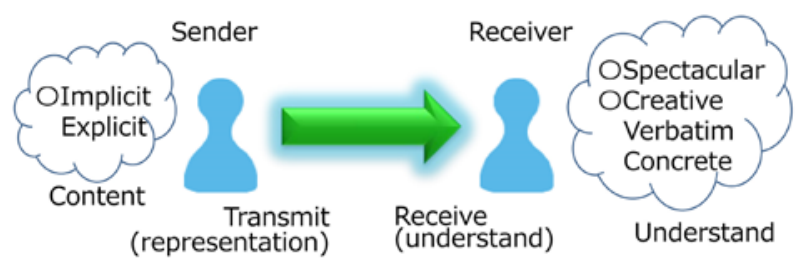

Fig.8 Affective communication

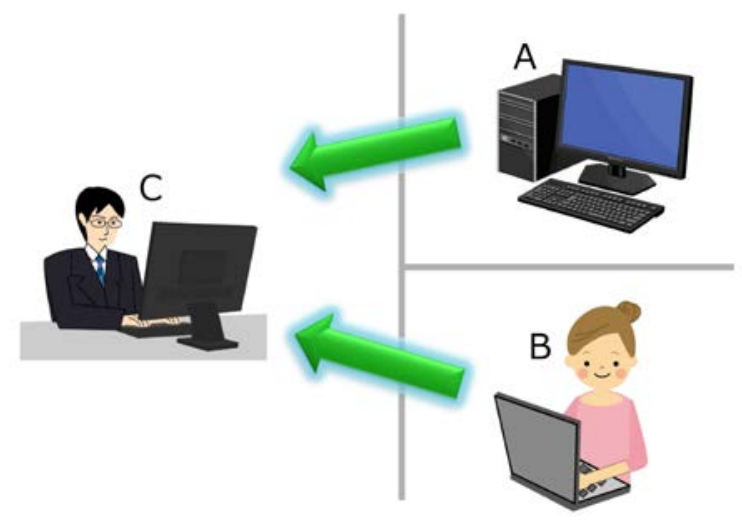

Fig.9 Kansei/Affective Turing test

Figure 9 shows the concept of the "Kansei/Affective Turing test”. In Fig. 9, it is assumed that the player C of the interrogator exchanges emotional communication with the computer-A, and the human player B, respectively. At this time, when the interrogator's player $\mathrm{C}$ cannot distinguish between the computer-A and the human $\mathrm{B}$, the computer $\mathrm{A}$ is said to have Kansei.

The "blurred portion" of the communication arrow in the "Kansei/Affective Turing test" is a Kansei/Affective expression, which is an important part full of "tacit knowledge".

The "blurred part" of the communication arrow includes elements of abduction and transilient in the inference process of "Innovation tetra" shown in Table 2.

Therefore, the realization of "Artificial Kansei” can be treated as a problem of how to realize the "blurred 
portion" of the arrow of Kansei/Affective communication in a computer. Since it is neither "deduction inference" nor "inductive inference", it will not be able to be realized with current AI technology computers.

\section{OPEN QUESTION IN FUTURE}

The "framework of the Kansei system" shown in Fig. 10 is proposed by Shiizuka [4], in which the vertical axis indicates "natural sensibility-artificial sensibility" and the horizontal axis indicates "measurement-expression".

One of the long-established researches on Kansei engineering is the modeling of natural kansei areas. However, we will construct an artificial Kansei methodology in the future so that we can understand "Kansei/Affective communication with ambiguous content". It is an area such as the tacit knowledge of a person included in the "blurred portion" shown in Fig.6.

We have described the basic concept of how to understand the "artificial Kansei/sensibility" that the authors usually have. Although there are many remaining issues in the future, it seems important to establish a cooperative relationship with artificial intelligence (AI) in areas that cannot be realized from artificial intelligence (AI) from a comprehensive perspective.

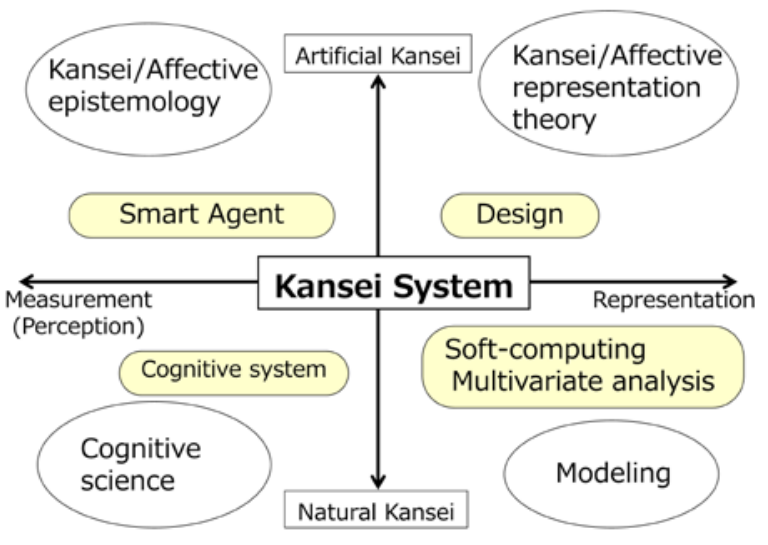

Fig. 10 Framework of Kansei system

Current artificial intelligence calculations, such as deep learning, are performed by "inductive operations" that learn from big data. Shiizuka [2] has shown that there are 88 inference processes generated from Innovation Tetra. It is a more general concept that encompasses today's AI technology approach.

Since mathematics is typical of deductive science, it will not be very difficult for computers to solve mathematical problems. Computers can also easily draw conclusions from large amounts of past data, so they are good at inductive operations.
Here the open question is presented. Can a computer perform abduction-like processing? Current computer algorithms will not be able to do abduction. In other words, the inference process implied by the innovation tetra proposed by Shiizuka[2] can be thought of as showing what the future computer should be.

Here we show one interest as shown in Fig.11. Newton discovered the law of universal gravitation from the fact that apples fell from trees in the garden. In other words, he noticed that apple and the earth were in tension. This is nothing but abduction. On the other hand, it can raise a very important question, "Can a computer perform abduction?” This means that we can't trust AI systems built on deep learning alone (Fig.11(b)). Therefore, Innovation Tetra's inference process seems to suggest a new way for AI [2]. Details will be appeared in the future.

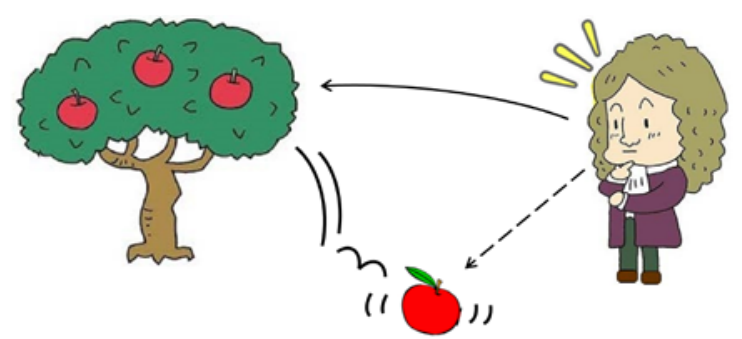

(a) Discovered the law of universal gravitation by abduction.

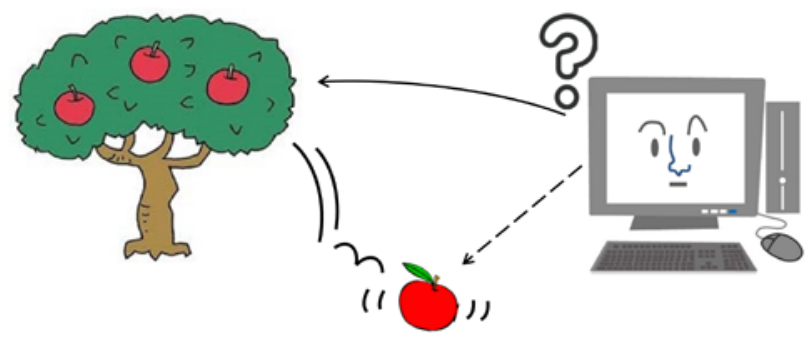

(b) Can a computer perform abduction?

Fig.11 It cannot be solved by deep learning alone!

\section{REFFERENCES}

[1] Hisao Shiizuka: Innovation Tetra - Toward the innovation engineering in Era of Kansei 3.0, Proceedings of 2015 Annual Conference of Japan Society of Kansei Engineering, H42, 2015

[2] Hisao Shiizuka: Framework of Innovation Engineering-“Play-Sensibility- Design” Drives Inference Process-, Proceedings of 2016 Spring Conference of Japan Society of Kansei Engineering, WD1-2, 2016

[3] Goodfellow et al. : Generative Adversarial Nets (2014) https://arxiv.org/abs/1406.2661

[4] Hisao Shiizuka: Kansei Engineering Hand Book, Asakura Shoten, 2013 